\title{
On the Construction of Gaussian Quadrature Rules from Modified Moments*
}

\author{
By Walter Gautschi
}

\begin{abstract}
Given a weight function $\omega(x)$ on $(\alpha, \beta)$, and a system of polynomials $\left\{p_{k}(x)_{\}_{k=0}}^{x}\right.$, with degree $p_{k}(x)=k$, we consider the problem of constructing Gaussian quadrature rules $\int_{x}^{\beta} f(x) \omega(x) d x \doteq \sum_{r=1}^{n} \lambda_{r}^{(n)} f\left(\xi_{r}^{\xi(n)}\right)$ from "modified moments" $v_{k}=\int_{x}^{\beta} p_{k}(x) \omega(x) d x$. Classical procedures take $p_{k}(x)=x^{k}$, but suffer from progressive ill-conditioning as $n$ increases. A more recent procedure, due to Sack and Donovan, takes for $\left\{p_{k}(x)\right\}$ a system of (classical) orthogonal polynomials. The problem is then remarkably well-conditioned, at least for finite intervals $[\alpha, \beta]$. In support of this observation, we obtain upper bounds for the respective asymptotic condition number. In special cases, these bounds grow like a fixed power of $n$. We also derive an algorithm for solving the problem considered, which generalizes one due to Golub and Welsch. Finally, some numerical examples are presented.
\end{abstract}

1. Introduction. Let $\omega(x)$ be a weight function on the (finite or infinite) interval $(\alpha, \beta)$, i.e., measurable and nonnegative on $(\alpha, \beta)$, with all moments

$$
\mu_{k}=\int_{\alpha}^{\beta} x^{k} \omega(x) d x, \quad k=0,1,2, \ldots,
$$

finite and $\mu_{0}>0$. Given a set $\left\{p_{k}(x)\right\}_{k=0}^{\infty}$ of polynomials, with degree $p_{k}=k$, we call

$$
v_{k}=\int_{\alpha}^{\beta} p_{k}(x) \omega(x) d x, \quad k=0,1,2, \ldots
$$

the modified moments of $\omega$. Clearly, $v_{k}=\mu_{k}$, if $p_{k}(x)=x^{k}$.

A Gaussian quadrature rule associated with the weight function $\omega$ is a functional

$$
G_{n} f=\sum_{r=1}^{n} \lambda_{r}^{(n)} f\left(\xi_{r}^{(n)}\right)
$$

which has the property that

$$
G_{n} f=\int_{\alpha}^{\beta} f(x) \omega(x) d x, \quad \text { all } f \in \mathbf{P}_{2 n-1},
$$

where $\mathbf{P}_{2 n-1}$ is the class of polynomials of degree $\leqq 2 n-1$. As is well known, $G_{n}$ exists uniquely for each $n=1,2,3, \ldots$ In fact, the abscissas $\xi_{r}^{(n)}$ are the zeros of $\pi_{n}(x)$,

Received October 13, 1969.

AMS Subject Classifications. Primary 6555, 6580; Secondary 3340.

Key Words and Phrases. Constructive theory of Gaussian quadrature rules, tables of Gaussian quadrature rules, numerical condition, orthogonal polynomials.

* Work supported by the Office of Naval Research (ONR) under grant N00014-67-A-0226-0011. 


$$
\pi_{n}\left(\xi_{r}^{(n)}\right)=0, \quad r=1,2, \ldots, n,
$$

where $\left\{\pi_{k}(x)\right\}_{k=0}^{\infty}$ is the system of orthonormal polynomials belonging to the weight function $\omega$,

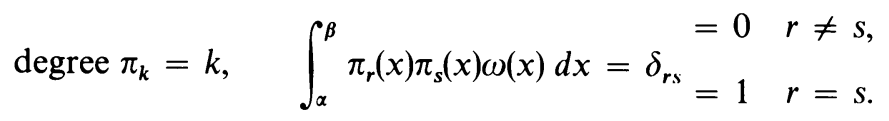

The weights $\lambda_{r}^{(n)}$, too, can be expressed in terms of these polynomials, e.g., by

$$
\lambda_{r}^{(n)}=\left(\sum_{k=0}^{n-1}\left[\pi_{k}\left(\xi_{r}^{(n)}\right)\right]^{2}\right)^{-1}, \quad r=1,2, \ldots, n,
$$

showing that $\lambda_{r}^{(n)}>0$.

The problem we want to consider is the computation of the functional $G_{n}$ (i.e., the computation of the abscissas $\xi_{r}^{(n)}$ and weights $\left.\lambda_{r}^{(n)}\right)$, given the modified moments $v_{k}$. In view of (1.5), (1.7), the problem may be considered as solved, once the orthonormal polynomials $\left\{\pi_{k}\right\}$ have been obtained accurately.

We note, incidentally, that these polynomials are also useful for the construction of rules

$$
\tilde{G}_{n} f=\tilde{\lambda}_{1}^{(n)} f(\xi)+\sum_{r=2}^{n} \tilde{\lambda}_{r}^{(n)} f\left(\tilde{\xi}_{r}^{(n)}\right)
$$

where $\xi$ is an arbitrary real number with $\pi_{n-1}(\xi) \neq 0$, and

$$
\tilde{G}_{n} f=\int_{\alpha}^{\beta} f(x) \omega(x) d x, \text { all } f \in \mathbf{P}_{2 n-2} .
$$

The abscissas $\hat{\xi}_{r}^{(n)}, r \geqq 2$, are then in fact the zeros (other than $\xi$ ) of

$$
\psi_{n}(x)=\pi_{n-1}(\xi) \pi_{n}(x)-\pi_{n}(\xi) \pi_{n-1}(x),
$$

while the weights $\tilde{\lambda}_{r}^{(n)}, r=1,2, \ldots, n$, are still given by the expression on the right of (1.7), if $\xi_{r}^{(n)}$ is replaced by $\tilde{\xi}_{r}^{(n)}$ and $\tilde{\xi}_{1}^{(n)}=\xi$ [2, Sections I.3-4].

The problem stated, in the special case $p_{k}(x)=x^{k}$, is classical, and a number of methods are known for its solution. However, the problem becomes increasingly illconditioned as $n$ increases, as we have shown in [6]. In order to obtain high-order Gaussian quadrature rules in this manner, it is therefore necessary to resort to multiple-precision computations. Alternatively, more elaborate procedures may be used, such as the one in [6], which do not rely on the moments $\mu_{k}$.

The general problem was considered recently by Sack and Donovan [12], in the case of polynomials $\left\{p_{k}\right\}$ satisfying a recurrence relation

$$
x p_{j}(x)=a_{j} p_{j+1}(x)+b_{j} p_{j}(x)+c_{j} p_{j-1}(x), \quad j=0,1,2, \ldots ; \quad p_{-1}(x)=0,
$$

with known coefficients $a_{j} \neq 0, b_{j}, c_{j}$. If $\left\{p_{k}\right\}$ are themselves orthogonal polynomials (e.g., Legendre polynomials, or Chebyshev polynomials of the first and second kind), and $(\alpha, \beta)$ is a finite interval, the results reported in [12] suggest that the problem is now remarkably well-conditioned. This new approach is therefore a useful alternative to the procedures mentioned above, in cases where the modified moments are accurately computable. 
It seems worthwhile, therefore, to investigate the condition of the general problem in the case of polynomials $\left\{p_{k}\right\}$ which are orthogonal with respect to some other weight function $w(x)$ on the interval $(a, b)$ [not necessarily equal to $(\alpha, \beta)$ ],

$$
\text { degree } p_{k}=k, \quad \int_{a}^{b} p_{r}(x) p_{s}(x) w(x) d x=0 \quad(r \neq s) .
$$

Such polynomials always satisfy a recurrence relation of the form (1.8). In Section 2 we obtain upper bounds for the condition number of this problem, relative to both finite and infinite intervals $(a, b)$. Asymptotic estimates of the condition number are given in Section 3 for certain classical weight functions on the interval $[-1,1]$. Rather strikingly, these estimates grow only like a fixed power of $n$. The condition is less favorable, in general, if the interval $(\alpha, \beta)$ is infinite. In Section 4 we then derive an algorithm for solving the problem under study. The algorithm reduces to one given by Golub and Welsch [8] for the case $p_{k}(x)=x^{k}$ and is similar (although not identical) to the algorithm of Sack and Donovan [12]. Some numerical examples are presented in Section 5. In particular, we obtain Gaussian quadrature rules $G_{n}$ for $n=1(1) 8,16,32$, relative to the weight functions

$$
\omega(x)=\frac{1}{2}\left(1+{ }_{\sin }^{\cos } m \pi x\right), \quad m=1(1) 12,
$$

on $[-1,1]$. Tables of the respective Gaussian abscissas and weights may be found on the microfiche card attached to this issue.

2. Condition of the Problem. In studying the condition of our problem, it is convenient to consider normalized modified moments as defined by

$$
\tilde{v}_{k}=h_{k}^{-1 / 2} \int_{\alpha}^{\beta} p_{k}(x) \omega(x) d x, \quad k=0,1,2, \ldots,
$$

where

$$
h_{k}=\int_{a}^{b} p_{k}^{2}(x) w(x) d x
$$

The normalized moments $\tilde{v}_{k}$ are invariant under different normalizations of the orthogonal polynomials $\left\{p_{k}\right\}$.

The problem stated in Section 1 is then equivalent to solving the system of $2 n$ (nonlinear) algebraic equations

$$
h_{k}^{-1 / 2} \sum_{r=1}^{n} \lambda_{r} p_{k}\left(\xi_{r}\right)=\tilde{v}_{k}, \quad k=0,1,2, \ldots, 2 n-1,
$$

for the unknowns $\lambda_{r}, \xi_{r}$. We can write these equations in vector form,

$$
F(y)=\tilde{v},
$$

by letting $y^{T}=\left[\lambda_{1}, \ldots, \lambda_{n}, \xi_{1}, \ldots, \xi_{n}\right], \quad \tilde{v}^{T}=\left[\begin{array}{lll}\tilde{v}_{0}, & \tilde{v}_{1}, \ldots, \tilde{v}_{2 n-1}\end{array}\right], F^{T}=$ $\left[F_{0}, F_{1}, \ldots, F_{2 n-1}\right]$, and

$$
F_{k}(y)=h_{k}^{-1 / 2} \sum_{r=1}^{n} \lambda_{r} p_{k}\left(\xi_{r}\right), \quad k=0,1, \ldots, 2 n-1 .
$$


In conformity with (1.3), we denote the solution of (2.4) by

$$
y_{0}^{T}=\left[\lambda_{1}^{(n)}, \ldots, \lambda_{n}^{(n)}, \xi_{1}^{(n)}, \ldots, \xi_{n}^{(n)}\right] .
$$

Given a vector norm $\|\cdot\|$, and an associated matrix norm, we may define, as in [6], a (relative) asymptotic condition number $\kappa_{n}$ for the problem (2.4), viz.

$$
\kappa_{n}=\frac{\|\tilde{v}\|}{\left\|y_{0}\right\|}\left\|\left[F_{y}\left(y_{0}\right)\right]^{-1}\right\|,
$$

where $F_{y}(y)$ denotes the Jacobian matrix of $F(y)$. An elementary computation shows that

$$
F_{y}\left(y_{0}\right)=H \Xi \Lambda
$$

where

(2.9) $\quad H=\operatorname{diag}\left(h_{0}^{-1 / 2}, h_{1}^{-1 / 2}, \ldots, h_{2 n-1}^{-1 / 2}\right), \quad \Lambda=\operatorname{diag}\left(1, \ldots, 1, \lambda_{1}, \ldots, \lambda_{n}\right)$

are diagonal matrices, and

$$
\left.\Xi=\mid \begin{array}{cccc}
p_{0}\left(\xi_{1}\right) \cdots & p_{0}\left(\xi_{n}\right) & p_{0}^{\prime}\left(\xi_{1}\right) \cdots & p_{0}^{\prime}\left(\xi_{n}\right) \\
p_{1}\left(\xi_{1}\right) \cdots & p_{1}\left(\xi_{n}\right) & p_{1}^{\prime}\left(\xi_{1}\right) \cdots & p_{1}^{\prime}\left(\xi_{n}\right) \\
\cdots \cdots & \cdots & \cdots & \cdots
\end{array}\right] .
$$

(For simplicity, we have written $\xi_{r}$ for $\xi_{r}^{(n)}$, and $\lambda_{r}$ for $\lambda_{r}^{(n)}$ in (2.9), (2.10).) Therefore,

$$
\kappa_{n} \leqq \frac{\|\tilde{v}\|}{\left\|y_{0}\right\|}\left\|\Lambda^{-1}\right\|\left\|\Xi^{-1} H^{-1}\right\| \text {. }
$$

For the following, it turns out to be convenient to work with the $L_{1}$-norm

$$
\|y\|_{1}=\sum_{k=0}^{2 n-1}\left|y_{k}\right|, \quad y^{T}=\left[y_{0}, y_{1}, \ldots, y_{2 n-1}\right] .
$$

THEOREM 2.1. Let $[a, b]$ be a finite interval. With $\left\{l_{\lambda}(x)\right\}_{\lambda=1}^{n}$ denoting the Lagrange interpolation polynomials associated with the abscissas $\left\{\xi_{r}^{\xi(n)}\right\}_{r=1}^{n}$,

$$
l_{\lambda}(x)=\prod_{v=1 ; v \neq \lambda}^{n} \frac{x-\xi_{v}^{(n)}}{\xi_{\lambda}^{(n)}-\xi_{v}^{\xi(n)}}=\frac{\pi_{n}(x)}{\pi_{n}^{\prime}\left(\xi_{\lambda}^{(n)}\right)\left(x-\xi_{\lambda}^{(n)}\right)},
$$

let

$$
\begin{aligned}
L_{n} & =\int_{a}^{b} \sum_{\lambda=1}^{n} l_{\lambda}^{2}(x) w(x) d x, \\
\sigma_{n} & =\max _{1 \leqq \lambda \leqq n}\left|l_{\lambda}^{\prime}\left(\xi_{\lambda}^{(n)}\right)\right| .
\end{aligned}
$$

Let, furthermore,

$$
\begin{aligned}
M_{\mu} & =\max _{a \leqq x \leqq b}\left|p_{\mu}(x)\right|, \quad h_{\mu}=\int_{a}^{b} p_{\mu}^{2}(x) w(x) d x, \\
\Delta_{n} & \left.=\max _{\{}\left|\xi_{\lambda}^{(n)}-x\right|: a \leqq x \leqq b, \lambda=1,2, \ldots, n\right\} .
\end{aligned}
$$


Then, using the $L_{1}$-norm (2.12) in (2.7), we have

$$
\kappa_{n} \leqq \kappa_{n}^{(1)} \kappa_{n}^{(2)} \kappa_{n}^{(3)}
$$

where

$\left(2.19^{1}\right) \quad \kappa_{n}^{(1)}=\max \left(1,1 / \min \lambda_{r}^{(n)}\right)\left[1+\left(2 \sigma_{n}+1\right) \Delta_{n}\right] /\left(\mu_{0}+\sum_{r=1}^{n}\left|\xi_{r}^{(n)}\right|\right)$,

$\left(2.19^{2}\right) \quad \kappa_{n}^{(2)}=\max _{0 \leqq \mu \leqq 2 n-1}\left(M_{\mu} / h_{\mu}^{1 / 2}\right)$,

$\left(2.19^{3}\right) \quad \kappa_{n}^{(3)}=L_{n}\|\tilde{v}\|_{1}$.

Remarks. 1. The quantity $\kappa_{n}^{(1)}$ depends only on the weight function $\omega(x)$, the quantity $\kappa_{n}^{(2)}$ only on $w(x)$, while $\kappa_{n}^{(3)}$ depends on both $\omega(x)$ and $w(x)$.

2. Normally, $[\alpha, \beta]=[a, b]$, in which case $\Delta_{n} \leqq b-a$.

Proof of Theorem 2.1. The key issue in the proof is a bound on the norm of $\Xi^{-1} H^{-1}$. We first determine $\Xi^{-1}$ explicitly.

Let

$$
\begin{aligned}
& P_{\lambda}(x)=l_{\lambda}^{2}(x)\left[1-2 l_{\lambda}^{\prime}\left(\xi_{\lambda}^{(n)}\right)\left(x-\xi_{\lambda}^{(n)}\right)\right], \\
& Q_{\lambda}(x)=l_{\lambda}^{2}(x)\left(x-\xi_{\lambda}^{(n)}\right)
\end{aligned}
$$

denote the fundamental Hermite interpolation polynomials belonging to the abscissas $\left\{\xi_{r}^{(n)}\right\}$. Let

$$
P_{\lambda}(x)=\sum_{\mu=0}^{2 n-1} a_{\lambda \mu} p_{\mu}(x), \quad Q_{\lambda}(x)=\sum_{\mu=0}^{2 n-1} b_{\lambda \mu} p_{\mu}(x) .
$$

Then, as in [4], one shows that

$$
\Xi^{-1}=\left[\begin{array}{l}
A \\
B
\end{array}\right], \quad A=\left[a_{\lambda \mu}\right], \quad B=\left[b_{\lambda \mu}\right] .
$$

By the orthogonality of $\left\{p_{k}(x)\right\}$, one obtains from (2.22)

$$
a_{\lambda \mu}=\frac{1}{h_{\mu}} \int_{a}^{b} P_{\lambda}(x) p_{\mu}(x) w(x) d x, \quad b_{\lambda \mu}=\frac{1}{h_{\mu}} \int_{a}^{b} Q_{\lambda}(x) p_{\mu}(x) w(x) d x,
$$

or, in view of (2.20), (2.21),

$$
a_{\lambda \mu}=\frac{1}{h_{\mu}}\left(\alpha_{\lambda \mu}-2 s_{\lambda}^{(n)} \beta_{\lambda \mu}\right), \quad b_{\lambda \mu}=\frac{1}{h_{\mu}} \beta_{\lambda \mu},
$$

where

$$
s_{\lambda}^{(n)}=l_{\lambda}^{\prime}\left(\xi_{\lambda}^{(n)}\right)
$$

and

$$
\alpha_{\lambda \mu}=\int_{a}^{b} l_{\lambda}^{2}(x) p_{\mu}(x) w(x) d x, \quad \beta_{\lambda \mu}=\int_{a}^{b} l_{\lambda}^{2}(x) p_{\mu}(x)\left(x-\xi_{\lambda}^{(n)}\right) w(x) d x .
$$

We are now in a position to bound the norm of $\Xi^{-1} H^{-1}$. From (2.26), we have 


$$
\begin{aligned}
& \sum_{\lambda=1}^{n}\left|\alpha_{\lambda \mu}\right| \leqq \sum_{\lambda=1}^{n} \int_{a}^{b} l_{\lambda}^{2}(x)\left|p_{\mu}(x)\right| w(x) d x \leqq M_{\mu} L_{n}, \\
& \sum_{\lambda=1}^{n}\left|\beta_{\lambda \mu}\right| \leqq \sum_{\lambda=1}^{n} \int_{a}^{b} l_{\lambda}^{2}(x)\left|p_{\mu}(x)\right|\left|x-\xi_{\lambda}^{(n)}\right| w(x) d x \leqq M_{\mu} \Delta_{n} L_{n} .
\end{aligned}
$$

Therefore, by (2.24),

$$
\sum_{\lambda=1}^{n}\left|a_{\lambda \mu}\right| \leqq \frac{M_{\mu}}{h_{\mu}}\left(1+2 \Delta_{n} \sigma_{n}\right) L_{n}, \quad \sum_{\lambda=1}^{n}\left|b_{\lambda \mu}\right| \leqq \frac{M_{\mu}}{h_{\mu}} \Delta_{n} L_{n} .
$$

Consequently,

$$
\left\|\Xi^{-1} H^{-1}\right\|_{1} \leqq\left[1+\left(2 \sigma_{n}+1\right) \Delta_{n}\right] L_{n} \max _{0 \leqq \mu \leqq 2 n-1} M_{\mu} / h_{\mu}^{1 / 2} .
$$

The theorem now follows from (2.11) and (2.29), by observing that

$$
\left\|y_{0}\right\|_{1}=\sum_{r=1}^{n}\left(\lambda_{r}^{(n)}+\left|\xi_{r}^{(n)}\right|\right)=\mu_{0}+\sum_{r=1}^{n}\left|\xi_{r}^{(n)}\right|,
$$

and

$$
\left\|\Lambda^{-1}\right\|_{1}=\max \left(1,1 / \min \lambda_{r}^{(n)}\right) \text {. }
$$

The following theorem is not restricted to finite intervals $[a, b]$.

THEOREM 2.2. Let $(a, b)$ be a finite or infinite interval. In addition to the notations of Theorem 2.1, let

$$
\begin{aligned}
L_{n, 1} & =\int_{a}^{b}\left[\sum_{\lambda=1}^{n} l_{\lambda}^{2}(x)\right]^{2} w(x) d x, \\
L_{n, 2} & =\int_{a}^{b}\left[\sum_{\lambda=1}^{n} l_{\lambda}^{2}(x)\left|x-\xi_{\lambda}^{(n)}\right|\right]^{2} w(x) d x .
\end{aligned}
$$

Then, using the $L_{1}$-norm (2.12) in (2.7), we have

$$
k_{n} \leqq k_{n}^{(1)} k_{n}^{(2)} \text {, }
$$

where

$$
\begin{aligned}
& k_{n}^{(1)}=\frac{\max \left(1,1 / \min \lambda_{r}^{(n)}\right)}{\mu_{0}+\sum_{r=1}^{n}\left|\xi_{r}^{(n)}\right|}, \\
& k_{n}^{(2)}=\left(L_{n, 1}^{1 / 2}+\left(1+2 \sigma_{n}\right) L_{n, 2}^{1 / 2}\right)\|\tilde{v}\|_{1} .
\end{aligned}
$$

Proof. The proof is virtually the same as that for Theorem 2.1, except that the sums in (2.27), (2.28) are estimated differently, using Schwarz's inequality:

$$
\begin{aligned}
\sum_{\lambda=1}^{n}\left|\alpha_{\lambda \mu}\right| & \leqq \int_{a}^{b} \sum_{\lambda=1}^{n} l_{\lambda}^{2}(x)\left|p_{\mu}(x)\right| w(x) d x \\
& \leqq\left\{\int_{a}^{b}\left[\sum_{\lambda=1}^{n} l_{\lambda}^{2}(x)\right]^{2} w(x) d x \int_{a}^{b} p_{\mu}^{2}(x) w(x) d x\right\}^{1 / 2}=h_{\mu}^{1 / 2} L_{n, 1}^{1 / 2},
\end{aligned}
$$




$$
\begin{aligned}
\sum_{\lambda=1}^{n}\left|\beta_{\lambda \mu}\right| & \leqq \int_{a}^{b} \sum_{\lambda=1}^{n} l_{\lambda}^{2}(x)\left|x-\xi_{\lambda}^{(n)}\right|\left|p_{\mu}(x)\right| w(x) d x \\
& \leqq\left\{\int_{a}^{b}\left[\sum_{\lambda=1}^{n} l_{\lambda}^{2}(x)\left|x-\xi_{\lambda}^{(n)}\right|\right]^{2} w(x) d x \int_{a}^{b} p_{\mu}^{2}(x) w(x) d x\right\}^{1 / 2}=h_{\mu}^{1 / 2} L_{n, 2}^{1 / 2} .
\end{aligned}
$$

Hence, as previously,

$$
\left\|\Xi^{-1} H^{-1}\right\|_{1} \leqq L_{n, 1}^{1 / 2}+\left(1+2 \sigma_{n}\right) L_{n, 2}^{1 / 2},
$$

and Theorem 2.2 follows from (2.11), (2.30), and (2.31).

For infinite intervals $(\alpha, \beta)$ the bounds in (2.18) and (2.33) are likely to be very large, even for only moderately large $n$, on account of the smallness of $\min \lambda_{r}^{(n)}$. Severe illconditioning, in such cases, is therefore a potential hazard. Example (iii) of Section 5 illustrates this point.

3. Asymptotic Estimates of Condition Number. We illustrate Theorem 2.1 of the previous section by considering some special (classical) weight functions. We use the notation

$$
a_{n} \dot{\sim} b_{n} \text { as } n \rightarrow \infty
$$

to express the fact that $\left|a_{n} / b_{n}\right|$ remains between positive bounds not depending on $n$, as $n \rightarrow \infty$.

THEOREM 3.1. Let $[a, b]=[\alpha, \beta]=[-1,1]$.

(a) If $\omega(x)=\left(1-x^{2}\right)^{\alpha},-\frac{1}{2} \leqq \alpha \leqq 0$, then $\kappa_{n}^{(1)} \leqq \bar{\kappa}_{n}^{(1)}$, where $\bar{\kappa}_{n}^{(1)} \dot{\sim} n^{2 \alpha+3}$ as $n \rightarrow \infty$.

(b) If $w(x)=(1-x)^{\alpha}(1+x)^{\beta}, \alpha>-1, \quad \beta>-1$, then, as $n \rightarrow \infty, \kappa_{n}^{(2)} \dot{\sim}$ $n^{q+1 / 2}$ if $q \geqq-\frac{1}{2}$, and $\kappa_{n}^{(2)} \dot{\sim} 1$ if $q<-\frac{1}{2}$, where $q=\max (\alpha, \beta)$.

(c) If $\omega(x)=w(x)$, then $\kappa_{n}^{(3)}=\mu_{0}^{3 / 2}$.

(d) If $\omega(x)=\left(1-x^{2}\right)^{\alpha},-1<\alpha \leqq 0$, and $w(x)=\left(1-x^{2}\right)^{\beta}, \quad \beta \geqq-\frac{1}{2}$, then $\kappa_{n}^{(3)} \leqq \bar{\kappa}_{n}^{(3)}$, where, as $n \rightarrow \infty, \bar{\kappa}_{n}^{(3)} \dot{\sim} n^{\beta+3 / 2}$ if $\alpha \neq 0$, and $\bar{\kappa}_{n}^{(3)} \dot{\sim} n^{\beta+7 / 2}$ if $\alpha=0 . * *$

Proof. (a) The polynomials $\pi_{k}(x)$, in this case, are the ultraspherical polynomials $P_{k}^{(\alpha, \alpha)}(x)$, properly normalized. We assume the zeros $\xi_{r}=\xi_{r}^{(n)}$ of $P_{n}^{(\alpha, \alpha)}(x)$ numbered in decreasing order,

$$
1>\xi_{1}>\xi_{2}>\cdots>\xi_{n}>-1 \text {. }
$$

They are symmetrically distributed with respect to the origin, i.e., $\xi_{r}=-\xi_{n+1-r}$. It is known $[14$, p. 121$]$ that for $|\alpha| \leqq \frac{1}{2}$,

$$
\cos \left(r \frac{\pi}{n+1}\right) \leqq \xi_{r} \leqq \cos \left(r-\frac{1}{2}\right) \frac{\pi}{n}, \quad r=1,2, \ldots,[n / 2] .
$$

From this one obtains by an elementary computation

$$
\cot \frac{\pi}{2(n+1)}-1 \leqq \sum_{r=1}^{n}\left|\xi_{r}\right| \leqq \frac{1}{\sin (\pi / 2 n)} .
$$

It follows that $\sum_{r=1}^{n}\left|\xi_{r}\right| \dot{\sim} n$, and therefore

**The result in the case $\alpha=0$ could be sharpened to read $\bar{\kappa}_{n}^{(3)} \dot{\sim} n^{\beta+5 / 2} \ln ^{2} n$. See footnote ${ }^{* * *}$. 


$$
\mu_{0}+\sum_{r=1}^{n}\left|\xi_{r}\right| \dot{\sim} n \quad \text { as } n \rightarrow \infty
$$

For the corresponding Christoffel numbers $\lambda_{r}^{(n)}$ we have [14, p. 350] $\min \lambda_{r}^{(n)}=$ $\lambda_{1}^{(n)}$, whenever $\alpha \geqq-\frac{1}{2}$. (Note that for $\alpha=-\frac{1}{2}$ all $\lambda_{r}^{(n)}$ are equal to $\pi / n$.) Moreover [14, p. 350], $\lambda_{1}^{(n)} \dot{\sim} n^{-2 \alpha-2}$. Therefore,

$$
\max \left(1,1 / \min \lambda_{r}^{(n)}\right) \dot{\sim} n^{2 \alpha+2} \text { as } n \rightarrow \infty .
$$

In order to estimate $\sigma_{n}$ in (2.15), we recall (see, e.g., $[11$, p. 63]) that for $\alpha \leqq 0$,

$$
v_{\lambda}(x)>|\alpha| \text { on }-1 \leqq x \leqq 1,
$$

where

$$
v_{\lambda}(x)=1-2 l_{\lambda}^{\prime}\left(\xi_{\lambda}\right)\left(x-\xi_{\lambda}\right) .
$$

We distinguish two cases, depending on whether $l_{\lambda}^{\prime}\left(\xi_{\lambda}\right) \geqq 0$ or $l_{\lambda}^{\prime}\left(\xi_{\lambda}\right)<0$. In the first case we let $x=1$ in (3.5), and obtain

$$
\left|l_{\lambda}^{\prime}\left(\xi_{\lambda}\right)\right|<\frac{1-|\alpha|}{2\left(1-\xi_{\lambda}\right)} .
$$

Using (3.1) and (3.2), we get

$$
\left|l_{\lambda}^{\prime}\left(\xi_{\lambda}\right)\right|<\frac{1-|\alpha|}{2\left(1-\xi_{1}\right)} \leqq \frac{1-|\alpha|}{2(1-\cos (\pi / 2 n))}=\frac{1-|\alpha|}{4 \sin ^{2}(\pi / 4 n)} .
$$

In the second case we let $x=-1$ in (3.5) and obtain by a similar reasoning

$$
\left|l_{\lambda}^{\prime}\left(\xi_{\lambda}\right)\right|<\frac{1-|\alpha|}{2\left(1+\xi_{\lambda}\right)} \leqq \frac{1-|\alpha|}{2\left(1+\xi_{n}\right)} \leqq \frac{1-|\alpha|}{4 \sin ^{2}(\pi / 4 n)} .
$$

Thus, in either case,

$$
\left|l_{\lambda}^{\prime}\left(\xi_{\lambda}\right)\right|<\frac{1-|\alpha|}{4 \sin ^{2}(\pi / 4 n)}
$$

and it follows that

$$
\sigma_{n}=\max _{\lambda}\left|l_{\lambda}^{\prime}\left(\xi_{\lambda}\right)\right| \leqq \bar{\sigma}_{n}, \quad \bar{\sigma}_{n} \dot{\sim} n^{2} \quad \text { as } n \rightarrow \infty .
$$

Combining (3.3), (3.4), and (3.6) gives the desired result.

(b) With $p_{\mu}(x)=P_{\mu}^{(\alpha, \beta)}(x)$, and $q=\max (\alpha, \beta)$, we have for the quantity $M_{\mu}$ in $(2.16)[14$, p. 166]

$$
M_{\mu}=\frac{\Gamma(\mu+q+1)}{\Gamma(q+1) \Gamma(\mu+1)} \dot{\sim} \mu^{q} \quad \text { if } q \geqq-\frac{1}{2},
$$

and

$$
M_{\mu} \dot{\sim} \mu^{-1 / 2} \quad \text { if } q<-\frac{1}{2} .
$$

Since 


$$
\begin{aligned}
& h_{\mu}^{1 / 2}=2^{(\alpha+\beta+1) / 2}\left[\frac{\Gamma(\mu+\alpha+1) \Gamma(\mu+\beta+1)}{(2 \mu+\alpha+\beta+1) \Gamma(\mu+1) \Gamma(\mu+\alpha+\beta+1)}\right]^{1 / 2} \dot{\sim} \mu^{-1 / 2}, \\
& \mu \rightarrow \infty,
\end{aligned}
$$

the assertion follows.

(c) Since $\omega(x)=w(x)$, we have $L_{n}=\mu_{0}$ (see, e.g., [11, p. 52]), and $\tilde{v}_{0}=\sqrt{ } \mu_{0}$, $\tilde{v}_{k}=0$ for $k>0$, giving $\kappa_{n}^{(3)}=\mu_{0}^{3 / 2}$ as asserted.

(d) In the ultraspherical case $\omega(x)=\left(1-x^{2}\right)^{\alpha},-1<\alpha \leqq 0$, it is known that $[14$, Problems 58, 60]

$$
\sum_{\lambda=1}^{n} l_{\lambda}^{2}(x) \leqq \frac{1}{|\alpha|} \quad(-1<\alpha<0), \quad \sum_{\lambda=1}^{n} l_{\lambda}^{2}(x) \leqq \frac{1}{\tan ^{2}(3 \pi / 4(2 n+1))} \quad(\alpha=0),
$$

uniformly on $[-1,1]$. Therefore,

$$
\begin{array}{lll}
L_{n} \leqq \bar{L}_{n}, & & (-1<\alpha<0), \\
& \bar{L}_{n}=m_{0} / \tan ^{2} \frac{3 \pi}{4(2 n+1)} & (\alpha=0),
\end{array}
$$

where $m_{0}=\int_{-1}^{1} w(x) d x$. In particular***,

$$
\begin{array}{ll}
\bar{L}_{n} \dot{\sim} 1 & (-1<\alpha<0), \\
\bar{L}_{n} \dot{\sim} n^{2} & (\alpha=0) .
\end{array}
$$

From (3.7), (3.9) (with $\alpha=\beta=q$ ) one finds by a simple computation that $M_{k} / h_{k}^{1 / 2}$ is an increasing function of $k$, if $\beta>-\frac{1}{2}$, and constant (for $k>0$ ) equal to $2 / \sqrt{ } \pi$, if $\beta=-\frac{1}{2}$. Therefore,

$$
\|\tilde{y}\|_{1}=\sum_{k=0}^{2 n-1}\left|\tilde{v}_{k}\right| \leqq \mu_{0} \sum_{k=0}^{2 n-1} M_{k} / h_{k}^{1 / 2} \leqq \mu_{0} 2 n M_{2 n-1} / h_{2 n-1}^{1 / 2},
$$

and using the asymptotic estimates in (3.7) and (3.9),

$$
\|\tilde{v}\|_{1} \leqq N_{n}, \quad N_{n} \dot{\sim} n^{\beta+3 / 2}
$$

The desired result now follows from (3.10)-(3.12). Theorem 3.1 is proved.

As an example, suppose we generate the Gaussian rule $G_{n}$ associated with the ultraspherical weight function $\left(1-x^{2}\right)^{\alpha},-\frac{1}{2} \leqq \alpha \leqq 0$, using as $\left\{p_{k}\right\}$ the ultraspherical polynomials with parameter $\beta,-\frac{1}{2} \leqq \beta \leqq 0$. Then Theorem 3.1, together with (2.18), tells us that the associated condition number $\kappa_{n}$ satisfies $\kappa_{n} \leqq \bar{\kappa}_{n}$, where, as $n \rightarrow \infty$,

$$
\begin{array}{ll}
\bar{\kappa}_{n} \dot{\sim} n^{2(\alpha+\beta)+5} & \text { if } \alpha \neq 0 \text { and } \alpha \neq \beta, \\
\bar{\kappa}_{n} \dot{\sim} n^{2 \beta+7} & \text { if } \alpha=0 \text { and } \beta \neq 0, \\
\bar{\kappa}_{n} \dot{\sim} n^{3 \alpha+7 / 2} & \text { if } \alpha=\beta .
\end{array}
$$

\footnotetext{
*** In the case $\alpha=0$, the sharper estimate $L_{n} \leqq L_{n}^{*}, L_{n}^{*} \dot{\sim} n \ln ^{2} n$ could be obtained by using an estimate for $\sum_{\lambda=1}^{n}\left|l_{\lambda}(x)\right|$, due to G. I. Natanson [10], in conjunction with the inequality $\sum_{\lambda=1}^{n} l_{\lambda}^{2}(x) \leqq$ $\left(\sum_{\lambda=1}^{n}\left|l_{\lambda}(x)\right|\right)^{2}$.
} 
Theorem 3.1, and the example just given, are presented here for the sole purpose of illustrating the magnitude of the condition number for the problem considered. It is not suggested that for such classical weight functions Gaussian quadrature rules be constructed from modified moments, since the respective orthogonal polynomials are explicitly known.

In practice, $\omega(x)$ being given, we have no control over $\kappa_{n}^{(1)}$. However, we may influence the magnitude of $\kappa_{n}^{(2)}$, and to some extent that of $\kappa_{n}^{(3)}$, by an appropriate choice of the polynomials $\left\{p_{k}\right\}$. In this connection, part (b) of Theorem 3.1 suggests the Chebyshev polynomials of the first kind, $p_{k}(x)=T_{k}(x)$, as both convenient and well-conditioned. With this choice, in fact, $\kappa_{n}^{(2)}=(2 / \pi)^{1 / 2}$.

4. An Algorithm for Generating Orthonormal Polynomials. We now derive an algorithm for generating the orthonormal polynomials $\left\{\pi_{k}(x)\right\}_{k=0}^{n}$ of (1.6), given a set of polynomials $\left\{p_{k}(x)\right\}$ (orthogonal or not), satisfying the recurrence relation

$$
\begin{aligned}
x p_{j}(x)^{n=} a_{j} p_{j+1}(x)+b_{j} p_{j}(x)+c_{j} p_{j-1}(x), \quad j=0,1,2, \ldots ; \\
p_{-1}(x)=0, \quad a_{j} \neq 0,
\end{aligned}
$$

and given the associated modified moments $\left\{v_{k}\right\}_{k=0}^{2 n}$ of (1.2). Our aim is toward determining the coefficients $\alpha_{j}, \beta_{j}(j=0,1,2, \ldots, n-1)$ in the recurrence relation

$$
x \pi_{j}(x)=\alpha_{j} \pi_{j+1}(x)+\beta_{j} \pi_{j}(x)+\alpha_{j-1} \pi_{j-1}(x), \quad j=0,1,2, \ldots ; \quad \pi_{-1}(x)=0 .
$$

We denote by

$$
(f, g)=\int_{x}^{\beta} f(x) g(x) \omega(x) d x
$$

the inner product with respect to which the $\pi_{k}(x)$ are orthonormal. Let $M=\left[m_{i j}\right]$ be the Gram matrix of order $n+1$, i.e.,

$$
m_{i j}=\left(p_{i}, p_{j}\right) \quad(i, j=0,1, \ldots, n) .
$$

Clearly, $M$ is positive-definite. Let

$$
M=R^{T} R, \quad R=\left[r_{i j}\right]
$$

be the Cholesky decomposition of $M$, and

$$
S=R^{-1}, \quad S=\left[s_{i j}\right] .
$$

Both $R$ and $S$ are upper triangular matrices with positive diagonal elements. By an observation of Mysovskih [9],

$$
\pi_{j}(x)=s_{0 j} p_{0}(x)+s_{1 j} p_{1}(x)+\cdots+s_{j j} p_{j}(x), \quad j=0,1, \ldots, n .
$$

Substituting (4.6) into (4.2), we can write

$$
\begin{aligned}
x\left[s_{0 j} p_{0}+\cdots+s_{j-1, j} p_{j-1}+s_{j j} p_{j}\right] & \\
=\alpha_{j}\left[s_{0, j+1} p_{0}+\cdots+s_{j, j+1} p_{j}+\right. & \left.s_{j+1, j+1} p_{j+1}\right]+\beta_{j}\left[s_{0 j} p_{0}+\cdots+s_{j j} p_{j}\right] \\
& +\alpha_{j-1}\left[s_{0, j-1} p_{0}+\cdots+s_{j-1, j-1} p_{j-1}\right] .
\end{aligned}
$$


Each term on the left, in view of (4.1), can be expressed as a linear combination of $p$ 's. Having done this, coefficients of equal p's must agree on both sides, because of the linear independence of the system $\left\{p_{k}(x)\right\}$. In particular, comparing the coefficients of $p_{j+1}$ and $p_{j}$, one gets

$$
s_{j j} a_{j}=\alpha_{j} s_{j+1, j+1}, \quad s_{j j} b_{j}+s_{j-1, j} a_{j-1}=\alpha_{j} s_{j, j+1}+\beta_{j} s_{j j},
$$

from which

$$
\alpha_{j}=\frac{s_{j j}}{s_{j+1, j+1}} a_{j}, \quad \beta_{j}=b_{j}-\frac{s_{j, j+1}}{s_{j+1, j+1}} a_{j}+\frac{s_{j-1, j}}{s_{j j}} a_{j-1} .
$$

Since

$$
\begin{aligned}
s_{j j} & =\frac{1}{r_{j j}} \quad(j=0,1, \ldots, n), \\
s_{j, j+1} & =-\frac{r_{j, j+1}}{r_{j j} r_{j+1, j+1}} \quad(j=0,1, \ldots, n-1),
\end{aligned}
$$

one finally obtains

$$
\begin{aligned}
& \alpha_{j}=\frac{r_{j+1, j+1}}{r_{j j}} a_{j}, \\
& j=0,1, \ldots, n-1 . \\
& \beta_{j}=b_{j}+\frac{r_{j, j+1}}{r_{j j}} a_{j}-\frac{r_{j-1, j}}{r_{j-1, j-1}} a_{j-1},
\end{aligned}
$$

For $j=0, r_{-1,0}$ is to be interpreted as zero, and $r_{-1,-1}$ as an arbitrary nonzero number.

We note that the formulas (4.7) reduce to those of Golub and Welsch [8], if $a_{j}=1$, $b_{j}=c_{j}=0$, i.e., $p_{k}(x)=x^{k}$. Also, of course, $M=R=I$, and thus $\alpha_{j}=a_{j}$, $\beta_{j}=b_{j}$, if $p_{k}(x)=\pi_{k}(x)$.

Once the Gram matrix $M$ is known, the desired coefficients $\alpha_{j}, \beta_{j}$ can thus be obtained from (4.7) by a Cholesky decomposition of $M$.

The Gram matrix $M$, on the other hand, can be built up from the modified moments $v_{j}$ in the following manner. Applying the recursion (4.1) twice, one has

$$
\begin{aligned}
m_{i j} & =\left(p_{i}, p_{j}\right)=\left(\frac{1}{a_{i-1}}\left[\left(x-b_{i-1}\right) p_{i-1}-c_{i-1} p_{i-2}\right], p_{j}\right) \\
& =\frac{1}{a_{i-1}}\left[\left(x p_{i-1}, p_{j}\right)-b_{i-1}\left(p_{i-1}, p_{j}\right)-c_{i-1}\left(p_{i-2}, p_{j}\right)\right] \\
& =\frac{1}{a_{i-1}}\left[\left(p_{i-1}, x p_{j}\right)-b_{i-1}\left(p_{i-1}, p_{j}\right)-c_{i-1}\left(p_{i-2}, p_{j}\right)\right] \\
& =\frac{1}{a_{i-1}}\left[a_{j}\left(p_{i-1}, p_{j+1}\right)+b_{j}\left(p_{i-1}, p_{j}\right)+c_{j}\left(p_{i-1}, p_{j-1}\right)-b_{i-1}\left(p_{i-1}, p_{j}\right)\right.
\end{aligned}
$$

that is, 


$$
m_{i j}=\frac{1}{a_{i-1}}\left[a_{j} m_{i-1, j+1}+\left(b_{j}-b_{i-1}\right) m_{i-1, j}+c_{j} m_{i-1, j-1}-c_{i-1} m_{i-2, j}\right]
$$

Since

$$
m_{-1, j}=0, \quad m_{0, i}=p_{0} v_{j} \quad(j=0,1, \ldots, 2 n),
$$

we have in (4.8) a recursive scheme to progressively build up the matrix $M$, using (4.9) as initial values.

The involvement of $v_{2 n}$ in (4.9) may appear puzzling at first, the Gaussian rule $G_{n}$ being determined uniquely by the first $2 n$ modified moments $v_{j}, j=0,1,2, \ldots, 2 n-1$. Actually, the role of $v_{2 n}$ is just that of normalizing $\pi_{n}(x)$, and its value affects neither $\xi_{r}^{\xi(n)}$ nor $\lambda_{r}^{(n)}$, in view of $(1.5),(1.7)$.

The algorithm presented here does not compare favorably with the algorithm of Sack and Donovan [12] in terms of speed and storage requirements. Our derivation, however, appears to us more transparent than the derivation given in [12].

5. Numerical Examples. All computations described in this section were carried out on the CDC 6500 computer in single precision arithmetic.

(i) We repeat and extend some of the experiments reported by Sack and Donovan [12]. For $p_{k}(x)$ we choose in turn $x^{k},(1+x)^{k}, P_{k}(x), T_{k}(x), U_{k}(x)$, where $P_{k}, T_{k}, U_{k}$ denote, respectively, the Legendre polynomial, and the Chebyshev polynomials of the first and second kind. We apply the algorithm of Section 4 to produce the coefficients $\alpha_{r}, \beta_{r}, r=0,1, \ldots, n-1$, in the recurrence relation for the normalized Legendre and Chebyshev polynomials, making use of the appropriate modified moments shown in Table 1. (Notations: $(2 n) ! !=2 \cdot 4 \cdots(2 n),(2 n+1) ! !=1 \cdot 3 \cdot 5 \cdots$ $(2 n+1), 0 ! !=(-1) ! !=1,(-3) ! !=-1$.

\section{TABLE 1. Modified moments $\mathrm{r}_{\mathrm{k}}$.}

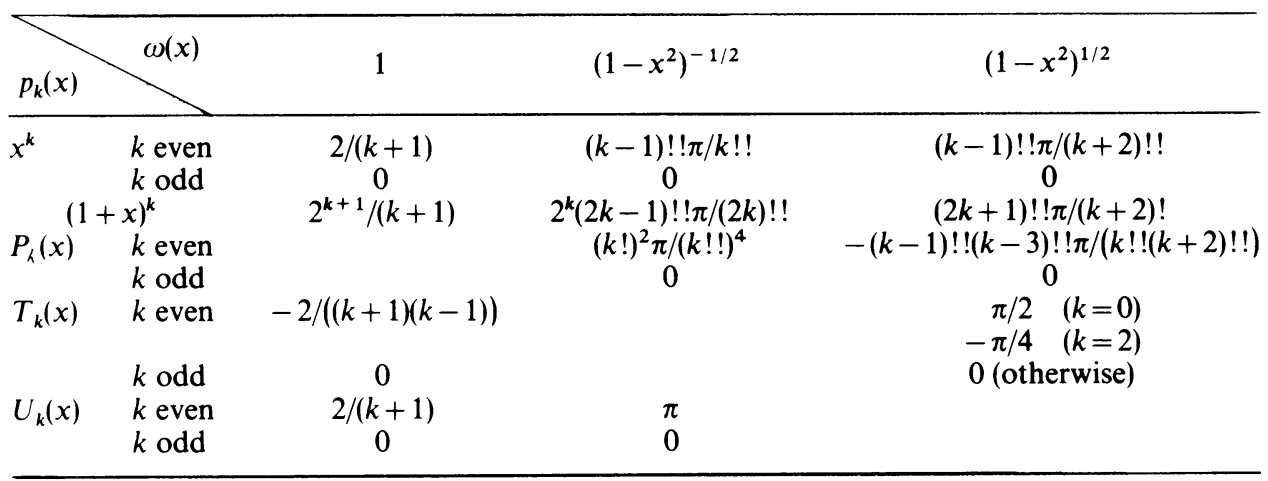

For the first two choices of $p_{k}(x)$, as is to be expected, the Gram matrix $M$ becomes increasingly ill-conditioned with $n$ increasing, and the Cholesky decomposition of $M$ eventually breaks down on taking the square root of a negative number. Prior to this, the errors in $\alpha_{r}$ and $\beta_{r}$ steadily increase, except for $\beta_{r}$ in the first case $\left\lceil p_{k}(x)=x^{k}\right\rceil$, where the algorithm consistently returns the correct value $\beta_{r}=0$. Sample values of 
errors are shown in Table 2 for the case $\omega(x)=1$. The Cholesky decomposition, in this case, fails at $n=23$ and $n=12$, respectively. The situation is very similar for the other two weight functions.

TABLE 2. Errors in the recursion coefficients for normalized Legendre polynomials.

\begin{tabular}{rccc}
\cline { 2 - 4 } & $p_{k}(x)=x^{k}$ & \multicolumn{2}{c}{$p_{k}(x)=(1+x)^{k}$} \\
\cline { 2 - 4 } & error in $\alpha_{r}$ & error in $\alpha_{r}$ & error in $\beta_{r}$ \\
\hline$r=5$ & $1.9 \times 10^{-12}$ & $1.8 \times 10^{-8}$ & $1.1 \times 10^{-8}$ \\
10 & $5.3 \times 10^{-9}$ & $2.3 \times 10^{-2}$ & $1.0 \times 10^{-1}$ \\
15 & $1.9 \times 10^{-5}$ & - & - \\
20 & $2.5 \times 10^{-2}$ & - & - \\
25 & - & - & - \\
\hline
\end{tabular}

No problems of any kind are encountered for the remaining three choices of $p_{k}(x)$, even going with $n$ as high as 100 . The coefficients $\alpha_{r}, \beta_{r}$ are obtained essentially to machine accuracy, the largest error observed being $7.1 \times 10^{-14}$.

(ii) Weight functions of interest in Fourier analysis are $\omega(x)=c_{m}(x)$, and $\omega(x)=$ $s_{m}(x)$, where

$$
\begin{aligned}
& c_{m}(x)=\frac{1}{2}(1+\cos m \pi x), \\
& s_{m}(x)=\frac{1}{2}(1+\sin m \pi x),
\end{aligned} \quad-1 \leqq x \leqq 1 ; \quad m=0,1,2, \ldots .
$$

Writing Fourier coefficients in the form [15]

$$
\begin{aligned}
\frac{1}{2 \pi} \int_{-\pi}^{\pi} f(x) \cos m x d x & =\int_{-1}^{1} f(\pi x) c_{m}(x) d x-\int_{-1}^{1} f(\pi x) s_{0}(x) d x \\
\frac{1}{2 \pi} \int_{-\pi}^{\pi} f(x) \sin m x d x=\int_{-1}^{1} f(\pi x) s_{m}(x) d x-\int_{-1}^{1} f(\pi x) s_{0}(x) d x, & \\
m & =1,2,3, \ldots,
\end{aligned}
$$

the first integrals on the right may be calculated by an appropriately weighted Gaussian quadrature rule, and the second integrals by classical Gaussian quadrature. To the best of our knowledge, no extensive tables exist for Gaussian rules $G_{n}$ associated with the weight functions (5.1). Admittedly, their usefulness is somewhat limited, because the set of points at which $f$ must be evaluated differs from one Fourier coefficient to another.

Our algorithm of Section 4 may be used to generate the required orthonormal polynomials. It is convenient to use it with $p_{k}(x)=P_{k}(x)$, since the modified moments can then be expressed in terms of spherical Bessel functions. In fact, using [1, p. 122] 


$$
\int_{-1}^{1} e^{i m \pi x} P_{k}(x) d x=i^{k}\left(\frac{2}{m}\right)^{1 / 2} J_{k+1 / 2}(m \pi)
$$

we obtain in the cosine-case

$$
v_{0}=1, \quad v_{2 k}=\frac{(-1)^{k}}{(2 m)^{1 / 2}} J_{2 k+1 / 2}(m \pi) \quad(k>0), \quad v_{2 k+1}=0 \quad(k \geqq 0),
$$

and in the sine-case

$$
v_{0}=1, \quad v_{2 k-1}=\frac{(-1)^{k+1}}{(2 m)^{1 / 2}} J_{2 k-1 / 2}(m \pi) \quad(k>0), \quad v_{2 k}=0 \quad(k>0) .
$$

To compute the Bessel functions in (5.3), (5.4), we use the procedure Japlusnt of [5], and the Gaussian abscissas and weights have been obtained using the relevant portions (both sequential and nonsequential) of Algorithm 331 [7]. The results are checked by having the quadrature rules regenerate the modified moments.

Table 3 of the microfiche section gives 12D values of $\xi_{r}^{(n)}, \pi \xi_{r}^{(n)}, \lambda_{r}^{(n)}$ for the Gaussian rule (1.3) associated with $\omega(x)=c_{m}(x)$, for $n=1(1) 8,16,32, m=1(1) 12$. (Because of symmetry, only the nonnegative abscissas and corresponding weights are listed.) Table 4 contains the analogous information for $\omega(x)=s_{m}(x), m=0(1) 12$.

(iii) To give an example for an infinite interval, we consider the "one-sided" Gauss-Hermite quadrature rules (1.3) corresponding to $\omega(x)=e^{-x^{2}}$ on $[0, \infty)$. Tables for such rules were recently published in [13], [3]. It seems natural, in this case, to choose $p_{k}(x)=H_{k}(x)$, the Hermite polynomials orthogonal with respect to $\omega(x)$ on $(-\infty, \infty)$. Then clearly,

$$
v_{0}=\sqrt{ } \pi / 2, \quad v_{2 k}=0 \quad(k>0) .
$$

To compute $v_{2 k+1}$, we start from the explicit representation

$$
H_{2 k+1}(x)=\sum_{r=0}^{k}(-1)^{r}\left(\begin{array}{c}
2 k+1 \\
2 r
\end{array}\right) \frac{(2 r) !}{2^{r} r !} 2^{2 k+1-r} x^{2 k+1-2 r} .
$$

Multiplying both sides by $e^{-x^{2}}$, and integrating between 0 and $x$, we obtain, in view of $\int_{0}^{\infty} e^{-x^{2}} x^{2 k+1-2 r} d x=\frac{1}{2}(k-r)$ !,

$$
v_{2 k+1}=\int_{0}^{\infty} e^{-x^{2}} H_{2 k+1}(x) d x=\sum_{r=0}^{k}(-1)^{r}\left(\begin{array}{c}
2 k+1 \\
2 r
\end{array}\right) \frac{(2 r) !}{2^{r} r !} 2^{2 k-r}(k-r) !,
$$

or, after simplification,

$$
v_{2 k+1}=\sum_{r=0}^{k} s_{r}^{(k)}, \quad s_{r}^{(k)}=2^{k}(-1)^{r} \prod_{i=r+1}^{k}(2 i) \prod_{i=k-r+1}^{k}(2 i+1) .
$$

Each $s_{r}^{(k)}$ being an integer, the sum in (5.6) can be evaluated in integer arithmetic without loss of accuracy, as long as no overflow occurs. Even so, however, it is found that the Gauss abscissas and weights obtained by our algorithm gradually deteriorate

† This procedure calls the gamma function $\Gamma(1+a)$. Since $a=1 / 2$ in our application, we have replaced "gamma $(1+a) "$ by its numerical value,$\pi / 2=.88622692545276$ in the procedure body. 
in accuracy. For $n=6$, for example, only 9-11 correct significant digits are obtained, while for $n=12$ only the first 2-4 significant digits are correct. It is believed that this deterioration of accuracy is a reflection of the progressive ill-conditioning of our problem. The quantity min $\lambda_{r}^{(n)}$, in fact, is about $9.8 \times 10^{-5}$ for $n=6$, and $1.2 \times 10^{-10}$ for $n=12$, resulting in a value of $k_{n}^{(1)}$ in (2.34) of the order $10^{4}$ and $10^{10}$, respectively.

Note Added in Proof. A substantially greater loss of accuracy is observed in Example (iii) if for $p_{k}(x)$ one chooses the Laguerre polynomials $L_{k}(x)$ instead of the Hermite polynomials $H_{k}(x)$. It is found that the Cholesky decomposition (4.4), in this case, breaks down for $n=7$, and the final results for $n=6$ are correct to only 3 decimal digits (using single precision arithmetic on the CDC 6500).

It is instructive to compare the condition number $\kappa_{n}$ for these two choices of the polynomials $p_{k}(x)$ on the basis of Theorem 2.2. The constant $k_{n}^{(1)}$ in (2.34) being the same for both choices of $p_{k}$, it suffices to compare $k_{n}^{(2)}$ in (2.35). Using the Gauss abscissas published in $\lceil 3\rceil$ to compute the Lagrange polynomials $l_{\lambda}(x)$, the quantities $L_{n, 1}$ and $L_{n, 2}$ in (2.32) may be evaluated by $2 n$-point Hermite quadrature (if $p_{k}=H_{k}$ ) and by $2 n$-point Gauss-Laguerre quadrature (if $p_{k}=L_{k}$ ). This will give $L_{n, 1}$ exactly (apart from rounding errors), and $L_{n, 2}$ at least approximately. For $n=6$, one obtains

$$
\begin{array}{lll}
L_{n, 1}=3.25 \times 10^{10}, & L_{n, 2}=3.80 \times 10^{11} & \left(p_{k}=H_{k}\right), \\
L_{n, 1}=1.72 \times 10^{19}, & L_{n, 2}=8.42 \times 10^{21} & \left(p_{k}=L_{k}\right) .
\end{array}
$$

Since $\|\tilde{v}\|_{1}$ is of comparable magnitude in both cases (approximately .808 for $p_{k}=H_{k}$, and 1.84 for $p_{k}=L_{k}$ ), one concludes that $k_{n}^{(2)}$ has the order of magnitude $10^{6}$ in the case of Hermite polynomials, but the order of magnitude $10^{11}$ in the case of Laguerre polynomials.

Purdue University

Lafayette, Indiana 47907 868

1. A. ERdélyi, Et AL., Tables of Integral Transforms. Vol. I, McGraw-Hill, New York, 1954. MR 15,

2. G. Freud, Orthogonale Polynome, Birkhăuser, Basel and Stuttgart, 1969.

3. D. Galant, "Gauss quadrature rules for the evaluation of $2 \pi^{-1 / 2} \int_{0}^{\infty} \exp \left(-x^{2}\right) f(x) d x$," Math. Comp., v. 23, 1969, p. 674.

4. W. GAUTSCHI, "On inverses of Vandermonde and confluent Vandermonde matrices. II," Numer. Math., v. 5, 1963, pp. 425-430. MR 29 \#1734.

5. W. GautsCHI, "Algorithm 236-Bessel functions of the first kind," Comm. ACM, v. 7, 1964, pp. 479-480.

6. W. GAUTSCHI, "Construction of Gauss-Christoffel quadrature formulas," Math. Comp., v. 22, 1968, pp. 251-270. MR 37 \#3755.

7. W. GAUTSCHI, "Algorithm 331-Gaussian quadrature formulas," Comm. ACM, v. 11, 1968, pp. $432-436$

8. G. H. Golub \& J. H. WelsCh, "Calculation of Gauss quadrature rules," Math. Comp., v. 23, 1969, pp. $221-230$.

9. I. P. MysovskiH, "On the construction of cubature formulas with the smallest number of nodes," Dokl. Akad. Nauk SSSR, v. 178, 1968, pp. 1252-1254 = Soviet Math. Dokl., v. 9, 1968, pp. 277-281. MR 36 \# 7328. 
10. G. I. Natanson, "A two-sided estimate for the Lebesgue function of the Lagrange interpolation process with Jacobi nodes," Izv. Vyš̌. Učebn. Zaved. Matematika, v. 1967, no. 11(66), pp. 67-74. (Russian)

11. I. P. Natanson, Constructive Function Theory, GITTL, Moscow, 1949; English transl., Vol. III: Interpolation and Approximation Quadratures, Ungar, New York, 1965. MR 11, 591; MR 33 \# 4529c. 12. R. A. SACK \& A. F. Donovan, An Algorithm for Gaussian Quadrature Given Generalized Moments, Department of Mathematics, University of Salford, Salford, England, 1969.

13. N. M. Steen, G. D. Byrne \& E. M. Gelbard, "Gaussian quadratures for the integrals $\int_{0}^{\infty} \exp \left(-x^{2}\right) f(x) d x$ and $\int_{0}^{b} \exp \left(-x^{2}\right) f(x) d x$," Math. Comp., v. 23, 1969, pp. 661-671.

14. G. Szegö, Orthogonal Polynomials, 2nd rev. ed., Amer. Math. Soc. Colloq. Publ., vol. 23, Amer. Math. Soc., Providence, R. I., 1959. MR 21 \# 5029.

15. I. ZAMFIRESCU, "An extension of Gauss' method for the calculation of improper integrals," Acad. R. P. Romine Stud. Cerc. Mat., v. 14, 1963, pp. 615-631. (Romanian) MR 32 \# 1906. 